\title{
The Impact of Depressive Symptoms on Neuropsychological Performance Tests in HIV-Infected Individuals: A Study of the Hawaii Aging with HIV Cohort
}

\author{
Sheri M. Shimizu ${ }^{1}$, Dominic C. Chow ${ }^{1 *}$, Victor Valcour ${ }^{1,2}$, Kamal Masaki ${ }^{3}$, Beau Nakamoto ${ }^{1,4}$, \\ Kalpana J. Kallianpur ${ }^{1}$, Cecilia Shikuma ${ }^{1}$ \\ ${ }^{1}$ Hawaii Center for AIDS, John A. Burns School of Medicine, University of Hawaii at Manoa, Honolulu, USA; ${ }^{2}$ Department of Neu- \\ rology and Division of Geriatric Medicine, Memory and Aging Center, Department of Medicine, University of California at San \\ Francisco, San Francisco, USA; ${ }^{3}$ Geriatric Medicine, John A. Burns School of Medicine, University of Hawaii, Honolulu, USA; \\ ${ }^{4}$ Department of Neurology, Straub Clinic and Hospital, Honolulu, USA. \\ E-mail: *dominicc@hawaii.edu
}

Received August 19 ${ }^{\text {th }}, 2011$; revised October $8^{\text {th }}, 2011$; accepted October $24^{\text {th }}, 2011$.

\begin{abstract}
Background: The frequency of neurocognitive impairment (NCI) in human immunodeficiency virus (HIV)-infected individuals remains high despite the availability of potent antiretroviral therapy (ART). The concurrence of depression among HIV-infected patients with NCI is common, especially among older individuals. Depression has been implicated as a risk factor for impaired neuropsychological performance (NP). This study explored the relationship between depressive symptoms and NP testing in HIV-infected individuals. Methods: A cross-sectional analysis was performed within the Hawaii Aging with HIV Cohort, a large prospective study of cognition of older (50 or more years old) compared to younger (20 to 39 years old) HIV-infected individuals. Results: Two hundred and eighty-five HIV infected participants (157 older and 128 younger) were administered a battery of NP tests to measure performance in major cognitive domains. Depressive symptoms were measured using the Beck Depression Inventory (BDI). The rates of depressive symptoms and neuropsychological impairment were similar in older and younger groups. Multivariate analyses revealed depressive symptoms were associated with NP test impairment in the younger group. In the older group, depressive symptoms were not associated with NP. Conclusion: This study suggests that depressive symptoms are associated with NP test impairment in younger HIV-infected individuals, but not in older individuals.
\end{abstract}

Keywords: Depression, HIV, Neurocognitive Function, Aging

\section{Background}

The frequency of neurocognitive impairment (NCI) in human immunodeficiency virus (HIV)-infected individuals remains high despite the availability of potent antiretroviral therapy (ART). Neuropsychological performance (NP) testing provides diagnostic clarification and grading of clinical severity for patients with subtle or obvious cognitive disorders. This testing provides clinical classification as to whether a HIV-infected patient may have HIV associated neurocognitive disorder (HAND) and dementia. Depression has been implicated as a risk factor for impaired NP in HIV individuals and has been shown to cause poor concentration, lack of in- terest and apathy towards NP testing [1-6]. Studies have found an association between subtypes of depressive symptoms and cognitive deficits [5,7]. However, inconsistencies are present with some studies demonstrating that depressive symptoms do not influence NP testing [8-12]. Few studies in the era of highly active antiretroviral therapy (HAART) have looked at depression in aging HIV populations.

Depression is commonly observed in HIV-infected individuals, where the prevalence is estimated to be over $36 \%$ [1,13]. Among older HIV-infected patients, depression is the second most prevalent behavioral health issues [14]. Aging is a potential risk factor for depression and 
neuropsychological impairment. Individuals with HIV today have a longer life expectancy with more than 114,951 individuals with AIDS over the age of 50 currently living in the United States [15]. There is limited data on neuropsychological functioning in older HIV infected individuals. Previous studies have found a higher frequency of dementia in older HIV infected individuals and that older adults may be at greater risk of NCI $[16,17]$. The importance of furthering our understanding of the impact of aging and depression on NP testing is necessitated by the growing population of older HIV infected individuals.

The changing nature of HIV treatment and longer patient survival highlight the need to re-evaluate these relationships in aging cohorts. This study evaluated the relationship between depressive symptoms using the Beck Depression Inventory (BDI) and NP tests in a wellcharacterized cohort of older and younger HIV-infected individuals.

\section{Methods}

\subsection{Study Population-Hawaii Aging with HIV Cohort}

The analyses utilized baseline (entry) data from the $\mathrm{Ha}$ waii Aging with HIV Cohort study (HAHC), a longitudinal cohort study assessing cognitive and neurologic outcomes in older compared to younger HIV-infected individuals [16]. Briefly, 158 older (50 years of age or older) and 128 younger (20 - 39 years of age) HIV-infected individuals were recruited between October 2001 to October 2005. Major exclusion criteria were the following: 1) head injury with loss of consciousness greater than 1 hour, 2) opportunistic brain infection, 3) learning disability, 4) major neurologic disease such as multiple sclerosis, major stroke, or current delirium, and 5) diagnosed major psychiatric disorder including bipolar illness, schizophrenia, or active major depression at baseline based on DSM-IV criteria. All individuals identified English as their primary language of communication. Most participants were Caucasian, and all were living in Hawaii at the time of enrollment and were recruited from all major islands of Hawaii.

\subsection{Neuropsychological Performance Tests}

Participant evaluations included the macro-neurologic examination, neuropsychological testing, medical intake with record of co-morbid illnesses, demographic data, risk behavior inventory, HIV laboratory parameters, and medication histories. The 80-minute neuropsychological battery designed to assess multiple cognitive domains most affected by HIV was administered and included the following: Choice and Sequential Reaction Time (CalCap), Rey Auditory Verbal Learning Test (RAVLT), Rey-Osterreith Complex Figure Test (RCF), Trail Making Tests A and B, WAIS-R Digit Symbol, Grooved Pegboard, Timed Gait, Odd Man Out, FAS, Animal Naming, Boston Naming Test (BNT), and WAIS-R Digit Span. The Human Subjects Review Committee of the University of Hawaii approved the study protocol, and all subjects signed a written informed consent prior to entry.

Raw scores from the neuropsychological tests were transformed to standardized $\mathrm{z}$-scores using published age- and education-matched normative datasets, as previously described [16]. Four composite neuropsychological indices were examined in the present study, including 1) Psychomotor (Grooved Pegboard Non-Dominant hand, Digit Symbol subtest from the WAIS-R, and Trail Making Test Trial B), 2) Memory (RAVLT Trial Delayed Recall Trial, Rey Complex Figure Test Delayed Recall, and the RAVLT Trial 5 minus Immediate Recall) 3) and a global NPZ-8 score. We used the NPZ8 score, a summary measure of neuropsychological testing performance and cognitive diagnostic categorizations for $\mathrm{NCI}$ and HIV-associated dementia as determined by a consensus conference with neurologists and neuropsychologists based on the American Academy of Neurology 1991 criteria [18]. The NPZ-8 score is defined as the average of z-scores for Timed Gait, Grooved Pegboard Dominant hand, Grooved Pegboard Non-Dominant hand, Trail Making Tests Parts A and B, the Digit Symbol subtest from the WAIS-R, and the Choice and Sequential Reaction Time trials from the CalCap test battery. This common composite score was first reported to relate to cognitive improvement following AZT treatment [19]. Our version substitutes the Choice and Sequential reaction times from the CalCap test for the Finger Tapping tests. For participants over the age of 60 , the NPZ-8 score did not include the CalCap tests due to limited normative data for this age group $(\mathrm{n}=18)$.

\subsection{Beck Depression Inventory}

The Beck Depression Inventory (BDI) was used to assess the presence and severity of depressive symptoms. The BDI has high validity and reliability in measuring depressive symptoms and takes approximately [20]. In this self-completed questionnaire, participants are asked to rate 21 items from zero to three according to how they have felt during the past week.

\subsection{Statistical Considerations}

All statistical analyses were carried out on SAS v9.1 (SAS Institute Inc., Cary, NC). Group differences in continuous outcome variables were analyzed using two- 
tailed t-tests. For discrete variables we used chi-square tests. We employed both simple correlation and regression analyses to assess the relationship between demographic factors and the cognitive endpoints. First, demographic predictors were tested by themselves using correlation analyses to determine whether they were associated with the cognitive endpoints. When significant associations were noted, the predictor was entered into the multiple regression analyses. BDI score was entered into all models. Associations were considered statistically significant if the p-value was less than 0.05 .

\section{Results}

There were 285 (157 older and 128 younger) HIV-infected participants enrolled. The study sample generally reflected the epidemiology of HIV infection in Hawaii exclusive of individuals in their forties, by study design, with the predominant risk factor for HIV being men who have sex with men, and a mean age of 54.5 years in the older group and 35.2 years in the younger group (Table 1). Caucasians comprised the majority of the participants in both the older group (69.6\%) and the younger group $(42.2 \%)$. There were no significant group differences between the younger and older participants in regards to CD4 count and percent on HAART therapy. The older group had a higher percentage of patients with undetect- able viral loads. Differences were noted in self- reported duration of infection $(p \leq 0.01)$, with a longer duration noted among older subjects. The older group also had a higher intelligence quotient (IQ) $(p \leq 0.01)$ and self-reported years of maximal educational attainment $(\mathrm{p} \leq$ $0.01)$. The younger group had a higher rate of self-reported substance abuse $(\mathrm{p} \leq 0.01)$. The mean total BDI score did not differ by age group, noted to be 8.6 in the older group and 9.1 in the younger group $(p=0.42)$. A summary of all demographic characteristics is presented in Table 1.

We completed a series of univariate analyses to determine if demographic characteristics correlated with the cognitive endpoints. The BDI, ethnicity, gender, IQ, and current CD4 count were associated with poor NP test and were included in the multivariate analyses. Viral load, antiretroviral therapy, years of HIV infection, and substance abuse were not associated with lower NP testing.

Results of multiple regression analyses testing the relative contribution of significant covariates and cognitive endpoints in the younger and older groups are summarized in Tables 2 and 3 respectively. In the younger group, the BDI score was significantly associated with lower NP performance in psychomotor speed $(p=0.04)$ and the NPZ-8 score $(p=0.03)$. In the older group BDI scores were not associated with cognitive endpoints,

Table 1. Baseline demographic and medical characteristics.

\begin{tabular}{|c|c|c|c|}
\hline Characteristics & Young & Old & $\mathbf{p}$ \\
\hline Sample Size & 128 & 157 & \\
\hline Age (years), Mean \pm SD & $35.2 \pm 4.9$ & $54.5 \pm 5.3$ & $<0.01$ \\
\hline$\%$ Male & 76.6 & 89.9 & $<0.01$ \\
\hline Education (years), Mean $\pm \mathrm{SD}$ & $13.2 \pm 1.9$ & $14.6 \pm 2.5$ & $<0.01$ \\
\hline \multicolumn{4}{|l|}{ Ethnicity } \\
\hline$\%$ Asian Pacific Islanders & 20.3 & 8.9 & \\
\hline$\%$ Caucasian & 42.2 & 69.6 & \\
\hline$\%$ Other & 37.5 & 21.5 & \\
\hline Intelligence Quotient score, Mean $\pm \mathrm{SD}$ & $103.6 \pm 6.2$ & $110.8 \pm 7.5$ & $<0.01$ \\
\hline Current CD4 Count cells $/ \mathrm{mm}^{3}$, Mean $\pm \mathrm{SD}$ & $435 \pm 228$ & $478 \pm 257$ & 0.14 \\
\hline$\%$ Undetectable Viral Load & 42.2 & 54.4 & 0.04 \\
\hline$\%$ on antiretroviral therapy & 76.8 & 68.5 & 0.13 \\
\hline Years Infected, Mean \pm SD & $7.18(5.6)$ & $11.8(5.31)$ & $<0.01$ \\
\hline$\%$ Substance Abuse-Current* & 22.6 & 8.8 & $<0.01$ \\
\hline Beck Depression Index (total score), Mean $\pm \mathrm{SD}$ & $9.1 \pm 7.2$ & $8.6 \pm 7.2$ & 0.42 \\
\hline
\end{tabular}

*Current substance abuse within last month including marijuana, cocaine, amphetamines, and heroin. 
Table 2. Multivariate analysis of neuropsychological performance tests to characteristics in younger HIV-infected individuals*.

\begin{tabular}{|c|c|c|c|c|c|c|}
\hline & \multicolumn{2}{|c|}{ Memory } & \multicolumn{2}{|c|}{ Psychomotor Speed $^{\mathrm{a}}$} & \multicolumn{2}{|c|}{ NPZ8 ${ }^{b}$} \\
\hline & $\beta$ & $\mathrm{p}$ & $\beta$ & $\mathrm{p}$ & $\beta$ & $\mathrm{p}$ \\
\hline Gender & & & & & -0.141 & 0.72 \\
\hline IQ & & & 0.028 & 0.06 & 0.027 & 0.02 \\
\hline Ethnicity & 0.031 & 0.75 & -0.08 & 0.53 & -0.095 & 0.31 \\
\hline CD4 count & 0.001 & 0.07 & 0.001 & 0.39 & 0.001 & 0.32 \\
\hline BDI & -0.001 & 0.5 & -0.054 & 0.04 & -0.053 & 0.03 \\
\hline
\end{tabular}

*All models included Beck Depression Index score (BDI), ethnicity, and CD4 count. Additional models included: ${ }^{\mathrm{a}} \mathrm{IQ} ;{ }^{\mathrm{b}}$ gender and IQ.

Table 3. Multivariate analysis of neuropsychological performance tests to characteristics in older HIV-infected individuals*.

\begin{tabular}{|c|c|c|c|c|c|c|}
\hline \multirow[b]{2}{*}{ Gender } & \multicolumn{2}{|c|}{ Memory } & \multicolumn{2}{|c|}{ Psychomotor Speed ${ }^{\mathrm{a}}$} & \multicolumn{2}{|c|}{ NPZ8 ${ }^{\mathrm{b}}$} \\
\hline & & & & & -0.22 & 0.08 \\
\hline IQ & & & 0.078 & $<0.01$ & 0.023 & $<0.01$ \\
\hline Ethnicity & -0.122 & 0.25 & -0.41 & $<0.01$ & -0.32 & $<0.01$ \\
\hline CD4 count & 0.001 & 0.04 & 0.023 & $<0.01$ & 0.025 & 0.31 \\
\hline BDI & 0.031 & 0.18 & -0.023 & 0.451 & -0.041 & 0.09 \\
\hline
\end{tabular}

*All models included Beck Depression Index score (BDI), ethnicity, and CD4 count. Additional models included: ${ }^{\mathrm{a} I Q}$; ${ }^{\mathrm{b}}$ gender and IQ.

however lower CD4 counts were associated with lower performance in psychomotor speed $(\mathrm{p}=<0.01)$ and memory $(p=0.04)$. In both groups, IQ was associated with NP tests.

When standardized clinical cut off points were applied to determine the severity of depressive symptoms (BDI < $10=$ minimal symptoms, BDI $10-16=$ mild symptoms, BDI $17-29=$ moderate symptoms, BDI $\geq 30=$ severe symptoms) on all subjects, significant trends were found with poorer cognitive performance on psychomotor and NPZ-8 scores (Figure 1). These trends were not seen in the memory score.

\section{Discussion}

The results of this study designed to determine the effects of depressive symptoms on neuropsychological performance in older and younger HIV infected individuals suggest that depressive symptoms are associated with neuropsychological impairment in younger HIV infected individuals. Neuropsychological impairment as evidenced by standardized composite z-scores of psychomotor speed and the previously defined NPZ-8 score was associated with depressive symptoms, however measures of disease progression such as CD4 count and viral load are not indicative of neuropsychological impairment in younger HIV infected individuals. This may be explained by the fact that the younger group had a shorter length of time to infection than the older group, resulting in less advanced disease progression and central nervous system (CNS) involvement than the older group. Depressive symptoms were associated neuropsychological impairment, especially in tests of psychomotor speed. This is consistent with previous studies in which depression has been implicated as a risk factor for impaired NP testingin HIV-infected individuals [2-6,21]. Depression remains predictive of poor treatment outcomes, decreased medication adherence, and increased mortality [22-24]. The younger group displayed higher rates of substance abuse, which when coupled with depressive symptoms may compound neuropsychological impairment in younger HIV infected individuals. Our findings highlight the need to identify depression early in young HIV infected individuals who exhibit neuropsychological impairment. Similar to the findings from the National Comorbidity Survey Replication Interventions on the general population, young adults are at high risk for anxiety and depression. Additionally, young adults with mental disorders are more likely to suffer disability compared to older individuals. This suggests that interventions aimed at prevention or early treatment of mental illness need to 


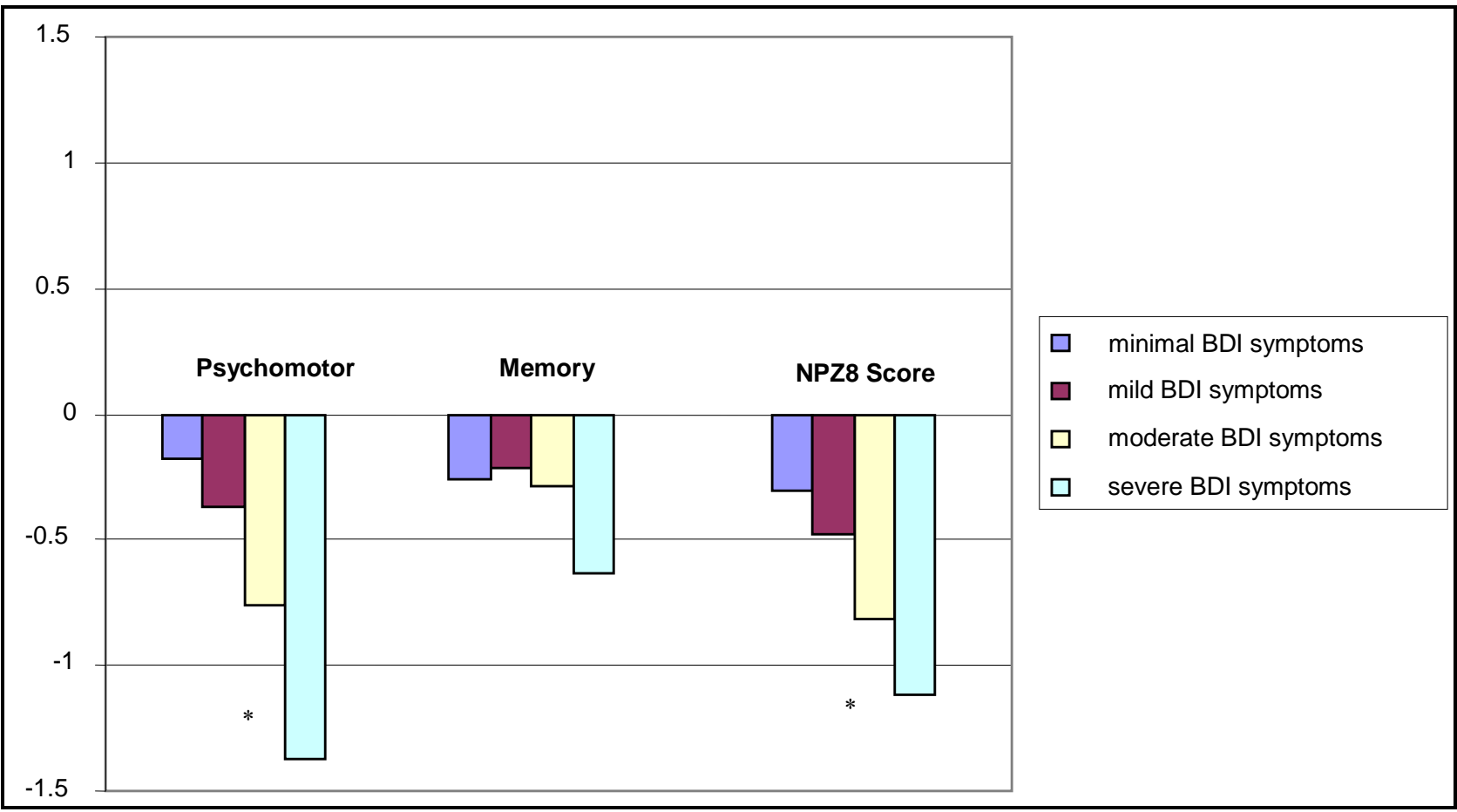

Figure 1. Neurocognitive impairment according to severity of depressive symptoms (units are in z-scores). *Significant trend, $\mathbf{p}<0.05$.

focus on youth and young adults [25].

The effect of depressive symptoms on neuropsychological impairment was not seen in the older group. Instead, our study suggests that neuropsychological decline may be a sequela of direct HIV related CNS involvement, as CD4 count was most predictive of neuropsychological impairment in the older group. It is known that HIV enters the CNS early in the course of infection and has been shown to produce detrimental effects on NP performance as the disease progresses. Impairment can range from mild cognitive and motor difficulties to AIDS related dementia and is consistent with frontal-subcortical pathology with evidence of decreased psychomotor processing speed, executive function, and memory [26,27]. In addition, aging has been associated with decreases in dopaminergic transmission as well as mitochondrial function and increases in oxidative free radicals [28-30]. These changes have also been seen in HIV infection $[31,32]$. It has been suggested that a more prominent relationship in each of these processes would be evidenced among older HIV-infected individuals [33].

There are several limitations of this study, primarily depressive symptoms were self-reported and measured at a single point in time. However, the BDI has previously shown construct validity in depressive populations [34]. Although individuals who were on antidepressant medications were included in this study, participants' were examined by a physician and determined that their symptoms were stable and without recent exacerbation. Although this was a cross-sectional study without controls, there was a significant trend in NCI and degree of depressive symptoms. There were differences between the racial groups between the young and old groups. However, there were no differences between BDI and NPZ-8 scores in regards to racial groups. This study may not have been powered to detect these differences. Additionally, the difference in the duration of HIV infection between age groups was significant, although there was no association between duration of HIV infection and NPZ-8 scores. No interaction between age groups and duration of HIV infection was noted. Our findings are consistent with a previous study that concluded that the duration of HIV illness and use of antiretroviral medication regimen were not related to depression [35].

The findings of this study emphasize the need for early evaluation of depressive symptoms particularly in younger HIV infected individuals. In older HIV infected individuals, cognitive status was related to CD4 count and attention to more aggressive antiretroviral treatment may be warranted.

\section{Acknowledgements}

This study was supported by the Hawaii Medical Student Aging Research National Training Center (National In- 
stitute on Aging, John A. Hartford Foundation and American Federation for Aging Research grant T35 AG-026722-02), National Institute of Neurological Disorders and Stroke (1U54NS43049). Additionally, D Chow received funding from the National Institutes of Health NHLBI 1 K23 HL088981, P20RR011091and NCRR 1 R25 RR019321. The content is solely the responsibility of the authors and does not necessarily represent the official views of the National Institute of Neurological Diseases and Stroke or the National Institutes of Health.

\section{REFERENCES}

[1] F. K. Judd and A. M. Mijch, "Depressive Symptoms in Patients with HIV Infection," The Australian and New Zealand Journal of Psychiatry, Vol. 30, No. 1, 1996, pp. 104-109. doi:10.3109/00048679609076077

[2] R. H. McAllister-Williams, I. N. Ferrier and A. H. Young, "Mood and Neuropsychological Function in Depression: The Role of Corticosteroids and Serotonin," Psychological Medicine, Vol. 28, No. 3, 1998, pp. 573-584. doi:10.1017/S0033291798006680

[3] T. D. Marcotte, R. K. Heaton, T. Wolfson, et al., "The Impact of HIV-Related Neuropsychological Dysfunction on Driving Behavior. The HNRC Group," Journal of the International Neuropsychological Society, Vol. 5, No. 7, 1999, pp. 579-592. doi:10.1017/S1355617799577011

[4] A. Ammassari, A. Antinori, M. S. Aloisi, et al., "Depressive Symptoms, Neurocognitive Impairment, and Adherence to Highly Active Antiretroviral Therapy among HIV-Infected Persons," Psychosomatics, Vol. 45, No. 5, 2004, pp. 394-402. doi:10.1176/appi.psy.45.5.394

[5] S. A. Castellon, D. J. Hardy, C. H. Hinkin, et al., "Components of Depression in HIV-1 Infection: Their Differential Relationship to Neurocognitive Performance," Journal of Clinical and Experimental Neuropsychology, Vol. 28, No. 3, 2006, pp. 420-437. doi:10.1080/13803390590935444

[6] J. D. Hartzell, I. E. Janke and A. C. Weintrob, "Impact of Depression on HIV Outcomes in the HAART Era," Journal of Antimicrobial Chemotherapy, Vol. 62, No. 2, 2008, pp. 246-255. doi:10.1093/jac/dkn193

[7] M. A. Cole, S. A. Castellon, A. C. Perkins, et al., "Relationship between Psychiatric Status and Frontal-Subcortical Systems in HIV-Infected Individuals," Journal of the International Neuropsychological Society, Vol. 13, No. 3, 2007, pp. 549-554. doi:10.1017/S135561770707066X

[8] R. A. Bornstein, H. A. Nasrallah, M. F. Para, C. C. Whitacre, P. Rosenberger and R. J. Fass, "Neuropsychological Performance in Symptomatic and Asymptomatic HIV Infection," AIDS, Vol. 7, No. 4, 1993, pp. 519-524. doi:10.1097/00002030-199304000-00011

[9] R. L. Mapou, W. A. Law, A. Martin, D. Kampen, A. M. Salazar and J. R. Rundell, "Neuropsychological Per- formance, Mood, and Complaints of Cognitive and Motor Difficulties in Individuals Infected with the Human Immunodeficiency Virus," Journal of Neuropsychiatry \& Clinical Neurosciences, Vol. 5, 1993, pp. 86-93.

[10] B. C. Bix, G. Glosser, W. Holmes, et al., "Relationship between Psychiatric Disease and Neuropsychological Impairment in HIV Seropositive Individuals," Journal of the International Neuropsychological Society, Vol. 1, No. 6, 1995, pp. 581-588. doi:10.1017/S1355617700000722

[11] K. J. Goggin, S. Zisook, R. K. Heaton, et al., "Neuropsychological Performance of HIV-1 Infected Men with Major Depression. HNRC Group. HIV Neurobehavioral Research Center," Journal of the International Neuropsychological Society, Vol. 3, 1997, pp. 457-464.

[12] S. B. Rourke, M. H. Halman and C. Bassel, "Neurocognitive Complaints in HIV-Infection and Their Relationship to Depressive Symptoms and Neuropsychological Functioning," Journal of Clinical and Experimental Neuropsychology, Vol. 21, No. 6, 1999, pp. 737-756. doi:10.1076/jcen.21.6.737.863

[13] E. G. Bing, M. A. Burnam, D. Longshore, et al., "Psychiatric Disorders and Drug Use among Human Immunodeficiency Virus-Infected Adults in the United States," Archives of General Psychiatry, Vol. 58, No. 8, 2001, pp. 721-728. doi:10.1001/archpsyc.58.8.721

[14] C. Grov, S. A. Golub, J. T. Parsons, M. Brennan and S. E. Karpiak, "Loneliness and HIV-Related Stigma Explain Depression among Older HIV-Positive Adults," AIDS Care, Vol. 22, No. 5, pp. 630-639. doi:10.1080/09540120903280901

[15] Centers for Disease Control and Prevention, "CDC Surveillance Report Notes Slowdown in New HIV/AIDS Rates," AIDS Policy Law, Vol. 17, No. 5, 2002, p. 8.

[16] V. Valcour, C. Shikuma, B. Shiramizu, et al., "Higher Frequency of Dementia in Older HIV-1 Individuals: The Hawaii Aging with HIV-1 Cohort," Neurology, Vol. 63, 2004, pp. 822-827.

[17] M. Cherner, R. J. Ellis, D. Lazzaretto, et al., "Effects of HIV-1 Infection and Aging on Neurobehavioral Functioning: Preliminary Findings," AIDS, Vol. 18, Suppl. 1, 2004, pp. S27-S34. doi:10.1097/00002030-200401001-00005

[18] American Academy of Neurology AIDS Task Force, "Nomenclature and Research Case Definitions for Neurologic Manifestations of Human Immunodeficiency Virus-Type 1 (HIV-1) Infection,” Neurology, Vol. 41, 1991, pp. 778-785.

[19] F. A. Schmitt, J. W. Bigley, R. McKinnis, P. E. Logue, R. W. Evans and J. L. Drucker, "Neuropsychological Outcome of Zidovudine (AZT) Treatment of Patients with AIDS and AIDS-Related Complex," The New England Journal of Medicine, Vol. 319, 1988, pp. 1573-1578. doi:10.1056/NEJM198812153192404

[20] A. T. Beck and R. A. Steer, "Internal Consistencies of the Original and Revised Beck Depression Inventory," Journal of Clinical Psychology 1984, Vol. 40, No. 6, pp. 
$1365-1367$. doi:10.1002/1097-4679(198411)40:6<1365::AID-JCLP22 70400615>3.0.CO;2-D

[21] F. K. Judd and A. M. Mijch, "Depressive Symptoms in Patients with HIV Infection," The Australian and New Zealand Journal of Psychiatry, Vol. 30, No. 1, 1996, pp. 104-109. doi:10.3109/00048679609076077

[22] T. Gibbie, A. Mijch, S. Ellen, et al., "Depression and Neurocognitive Performance in Individuals with HIV/ AIDS: 2-Year Follow-Up," HIV Medicine, Vol. 7, No. 2, 2006, pp. 112-121. doi:10.1111/j.1468-1293.2006.00350.x

[23] F. K. Judd, A. M. Cockram, A. Komiti, A. M. Mijch, J. Hoy and R. Bell, "Depressive Symptoms Reduced in Individuals with HIV/AIDS Treated with Highly Active Antiretroviral Therapy: A Longitudinal Study," The Australian and New Zealand Journal of Psychiatry, Vol. 34, No. 6, 2000, pp. 1015-1021. doi:10.1080/000486700278

[24] C. H. Hinkin, S. A. Castellon, R. S. Durvasula, et al., "Medication Adherence among HIV+ Adults: Effects of Cognitive Dysfunction and Regimen Complexity," Neurology, Vol. 59, 2002, pp. 1944-1950.

[25] R. C. Kessler, P. Berglund, O. Demler, R. Jin, K. R. Merikangas and E. E. Walters, "Lifetime Prevalence and Age-of-Onset Distributions of DSM-IV Disorders in the National Comorbidity Survey Replication," Archives of General Psychiatry, Vol. 62, No. 6, 2005, pp. 593-602. doi:10.1001/archpsyc.62.6.593

[26] L. Boisse, M. J. Gill and C. Power, "HIV Infection of the Central Nervous System: Clinical Features and Neuropathogenesis," Neurologic Clinics, Vol. 26, No. 3, 2008, pp. 799-819. doi:10.1016/i.ncl.2008.04.002

[27] B. M. Ances and R. J. Ellis, "Dementia and Neurocognitive Disorders Due to HIV-1 Infection," Seminars in Neurology, Vol. 27, No. 1, 2007, pp. 86-92. doi:10.1055/s-2006-956759

[28] D. B. Miller, J. P. O'Callaghan and S. F. Ali, “Age as a Susceptibility Factor in the Striatal Dopaminergic Neuro- toxicity Observed in the Mouse Following Substituted Amphetamine Exposure," Annals of the New York Academy of Sciences, Vol. 914, 2000, pp. 194-207. doi:10.1111/j.1749-6632.2000.tb05196.x

[29] V. A. Bohr, "Repair of Oxidative DNA Damage in Nuclear and Mitochondrial DNA, and Some Changes with Aging in Mammalian Cells," Free Radical Biology and Medicine, Vol. 32, No. 9, 2002, pp. 804-812. doi:10.1016/S0891-5849(02)00787-6

[30] J. B. Lohr, "Oxygen Radicals and Neuropsychiatric Illness. Some Speculations," Archives of General Psychiatry, Vol. 48, No. 12, 1991, pp. 1097-1106.

[31] J. R. Berger and G. Arendt, "HIV Dementia: The Role of the Basal Ganglia and Dopaminergic Systems," Journal of Psychopharmacology, Vol. 14, No. 3, 2000, pp. 214221. doi: $10.1177 / 026988110001400304$

[32] T. Yamaguchi, I. Katoh and S. Kurata, "Azidothymidine Causes Functional and Structural Destruction of Mitochondria, Glutathione Deficiency and HIV-1 Promoter Sensitization," European Journal of Biochemistry, Vol. 269, No. 11, 2002, pp. 2782-2788. doi:10.1046/j.1432-1033.2002.02954.x

[33] F. L. Wilkie, K. Goodkin, I. Khamis, et al., "Cognitive Functioning in Younger and Older HIV-1-Infected Adults," Journal of Acquired Immune Deficiency Syndromes, Vol. 33, Suppl. 2, 2003, pp. S93-S105. doi:10.1097/00126334-200306012-00006

[34] C. K. Schotte, M. Maes, R. Cluydts, D. De Doncker and P. Cosyns, "Construct Validity of the Beck Depression Inventory in a Depressive Population," Journal of Affective Disorders, Vol. 46, No. 2, 1997, pp. 115-125. doi:10.1016/S0165-0327(97)00094-3

[35] A. Komiti, F. Judd, P. Grech, et al., "Depression in People Living with HIV/AIDS Attending Primary Care and Outpatient Clinics," The Australian and New Zealand Journal of Psychiatry, Vol. 37, 2003, pp. 70-77. doi:10.1046/j.1440-1614.2003.01118.x 